\title{
Structural Change in a Multi-Sector Model of Growth
}

\author{
L Rachel Ngai Christopher A Pissarides* \\ November 2004 (this revision, May 2006, forthcoming in the \\ American Economic Review)
}

\begin{abstract}
We study a multi-sector model of growth with differences in TFP growth rates across sectors and derive sufficient conditions for the coexistence of structural change, characterized by sectoral labor reallocation, and balanced aggregate growth. The conditions are weak restrictions on the utility and production functions. Along the balanced growth path, labor employed in the production of consumption goods gradually moves to the sector with the lowest TFP growth rate, until in the limit it is the only sector with nontrivial employment of this kind. The employment shares of intermediate and capital goods remain constant during the reallocation process. (JEL O41, O14, E29)
\end{abstract}

Economic growth takes place at uneven rates across different sectors of the economy. This paper has two objectives related to this fact: (a) to derive the implications of different sectoral total factor productivity (TFP) growth rates for structural change, the name given to the shifts in industrial

${ }^{*}$ Ngai: Centre for Economic Performance, London School of Economics and CEPR (e-mail: l.ngai@lse.ac.uk). Pissarides: Centre for Economic Performance, London School of Economics, CEPR and IZA (e-mail: c.pissarides@lse.ac.uk). We have benefited from comments received at several presentations (the CEPR ESSIM 2004 meetings, the SED 2004 annual conference, the NBER 2004 Summer Institute, the 2004 Canadian Macroeconomic Study Group and several universities), and from Fernando Alvarez, Francesco Caselli, Antonio Ciccone, Nobu Kiyotaki, Robert Lucas, Nick Oulton, Danny Quah, Sergio Rebelo, Robert Shimer, Nancy Stokey, Richard Rogerson, Jaume Ventura and two anonymous referees. Funding from the CEP, a designated ESRC Research Centre, is acknowledged. 
employment shares that take place over long periods of time, and (b) to show that even with ongoing structural change, the economy's aggregate ratios can be constant. We refer to the latter as aggregate balanced growth. The restrictions needed to yield structural change consistent with the facts and aggregate balanced growth are weak restrictions on functional forms that are frequently imposed by macroeconomists in related contexts.

We obtain our results in a baseline model of many consumption goods and a single capital good, supplied by a sector that we label manufacturing. Our baseline results are consistent with the existence of intermediate goods and many capital goods under some reasonable restrictions. Production functions in our model are identical in all sectors except for their rates of TFP growth and each sector produces a differentiated good that enters a constant elasticity of substitution (CES) utility function. We show that a low (below one) elasticity of substitution across final goods leads to shifts of employment shares to sectors with low TFP growth. In the limit the employment share used to produce consumption goods vanishes from all sectors except for the one with the smallest TFP growth rate, but the employment shares used to produce capital goods and intermediate goods converge to non-trivial stationary values. If the utility function in addition has unit inter-temporal elasticity of substitution, during structural change the aggregate capital-output ratio is constant and the aggregate economy is on a balanced growth path.

Our results contrast with the results of Cristina Echevarria (1997), John Laitner (2000), Francesco Caselli and Wilbur Coleman II (2001) and Douglas Gollin, Stephen Parente and Richard Rogerson (2002) who derived structural change in a two- or three-sector economy with non-homothetic preferences. Our results also contrast with the results of Piyabha Kongsamut, Sergio Rebelo and Danyang Xie (2001) and Reto Foellmi and Josef Zweimuller (2005), who derived simultaneous constant aggregate growth and structural change. Kongsamut et al. (2001) obtain their results by imposing a restriction that maps some of the parameters of their Stone-Geary utility function onto the parameters of the production functions, abandoning one of the most useful conventions of modern macroeconomics, the complete independence of preferences and technologies. Foellmi and Zweimuller (2005) obtain their results by assuming endogenous growth driven by the introduction of new goods into a hierarchic utility function. Our restrictions are quantitative restrictions on a conventional CES utility function that maintains the independence of the parameters of preferences and technologies. 
Our results confirm William J Baumol's (1967) claims about structural change. Baumol divided the economy into two sectors, a "progressive" one that uses new technology and a "stagnant" one that uses labor as the only input. He then claimed that the production costs and prices of the stagnant sector should rise indefinitely, a process known as "Baumol's cost disease," and labor should move in the direction of the stagnant sector. ${ }^{1}$

In the more recent empirical literature two competing explanations (which can coexist) have been put forward for structural change. Our explanation, which is sometimes termed "technological" because it attributes structural change to different rates of sectoral TFP growth, and a utilitybased explanation, which requires different income elasticities for different goods and can yield structural change even with equal TFP growth in all sectors. Baumol, Sue Anne Batey Blackman and Edward N. Wolff (1985) provide empirical evidence at the 2-digit industry level, consistent with our model. Irving B. Kravis, Alan W. Heston and Robert Summers (1983) also present evidence that favors the technological explanation, at least when the comparison is between manufacturing and services. Two features of their data that are satisfied by the technological explanation proposed in this paper are (a) relative prices reflect differences in TFP growth rates and (b) real consumption shares vary a lot less over time than nominal consumption shares. ${ }^{2}$ Our model is also consistent with the observed positive correlation between employment growth and relative price inflation across two-digit sectors ${ }^{3}$ and with historical OECD evidence presented by Simon Kuznets (1966) and Angus Maddison (1980) for one-digit sectors. ${ }^{4}$

\footnotetext{
${ }^{1}$ Baumol controversially also claimed that as more weight is shifted to the stagnant sector, the economy's growth rate will be on a declining trend and eventually converge to zero. This claim contrasts with our finding that the economy is on a balanced-growth path. We get our result because we include capital in our analysis, ironically left out of the analysis by Baumol (1967, p.417) "primarily for ease of exposition ... that is [in] essential to the argument".

${ }^{2}$ See Rodney E. Falvey and Norman Gemmell (1996) for an update of some of their results. Falvey and Gemmell find a unit income elasticity and a small (negative) price elasticity for services in a cross-section of countries, consistent with our results.

${ }^{3}$ These correlations are shown in the working paper version of this paper, L. Rachel Ngai and Christopher A. Pissarides (2004).

${ }^{4}$ Kuznets (1966) documented structural change for 13 OECD countries and the USSR between 1800 and 1960 and Maddison (1980) documented the same pattern for 16 OECD countries from 1870 to 1987 . They both found a pattern with the same general features as the predictions that we obtain when the ranking of the average historical TFP growth rates is agriculture followed by manufacturing followed by services.
} 
Section 1 describes our model of growth with many sectors and sections 2 and 3 respectively derive the conditions for structural change and balanced aggregate growth. In sections 4 and 5 we study two extensions of our baseline model, one where consumption goods can also be used as intermediate inputs and one where there are many capital goods. The Appendix discusses the implications of one more extension, differences in capital intensities across sectors, and contains proofs of the main results.

\section{An economy with many sectors}

The baseline economy consists of an arbitrary number of $m$ sectors. Sectors $i=1, \ldots, m-1$ produce only consumption goods. The last sector, which is denoted by $m$ and labeled manufacturing, produces both a final consumption good and the economy's capital stock. We derive the equilibrium as the solution to a social planning problem. The objective function is

$$
U=\int_{0}^{\infty} e^{-\rho t} v\left(c_{1}, . ., c_{m}\right) d t
$$

where $\rho>0, c_{i} \geq 0$ are per-capita consumption levels and the instantaneous utility function $v($.$) is concave and satisfies the Inada conditions.$ The constraints of the problem are as follows.

The labor force is exogenous and growing at rate $\nu$ and the aggregate capital stock is endogenous and defines the state of the economy. Sectoral allocations are controls that satisfy

$$
\sum_{i=1}^{m} n_{i}=1 ; \quad \sum_{i=1}^{m} n_{i} k_{i}=k
$$

where $n_{i} \geq 0$ is the employment share and $k_{i} \geq 0$ is the capital-labor ratio in sector $i$, and $k \geq 0$ is the aggregate capital-labor ratio. There is free mobility for both factors.

All production in sectors $i=1, \ldots, m-1$ is consumed but in sector $m$ production may be either consumed or invested. Therefore:

$$
\begin{aligned}
c_{i} & =F^{i}\left(n_{i} k_{i}, n_{i}\right) \quad \forall i \neq m \\
\dot{k} & =F^{m}\left(n_{m} k_{m}, n_{m}\right)-c_{m}-(\delta+\nu) k
\end{aligned}
$$

where $\delta>0$ is the depreciation rate. Production function $F^{i}(.,$.$) has$ constant return to scale, positive and diminishing returns to inputs, and satisfies the Inada conditions. 
The social planner chooses the allocation of factors $n_{i}$ and $k_{i}$ across $m$ sectors through a set of static efficiency conditions,

$$
v_{i} / v_{m}=F_{K}^{m} / F_{K}^{i}=F_{N}^{m} / F_{N}^{i} \quad \forall i
$$

The allocation of output to consumption and capital is chosen through a dynamic efficiency condition,

$$
-\dot{v}_{m} / v_{m}=F_{K}^{m}-(\delta+\rho+\nu) .
$$

where $F_{N}^{i}$ and $F_{K}^{i}$ are the marginal products of labor and capital in sector $i .^{5}$ By (5), the rates of return to capital and labor are equal across sectors.

In order to focus on the implications of different rates of TFP growth across sectors we assume production functions are identical in all sectors except for their rates of TFP growth:

$$
F^{i}=A_{i} F\left(n_{i} k_{i}, n_{i}\right) ; \quad \dot{A}_{i} / A_{i}=\gamma_{i} ; \quad \forall i
$$

With these production functions, we show in the Appendix that static efficiency and the resource constraints (2) imply

$$
k_{i}=k ; \quad p_{i} / p_{m}=v_{i} / v_{m}=A_{m} / A_{i} ; \quad \forall i
$$

where $p_{i}$ is the price of good $i$ in the decentralized economy.

The utility function has constant elasticities both across goods and over time:

$$
v\left(c_{1}, \ldots, c_{m}\right)=\frac{\phi(.)^{1-\theta}-1}{1-\theta} ; \quad \phi(.)=\left(\sum_{i=1}^{m} \omega_{i} c_{i}^{(\varepsilon-1) / \varepsilon}\right)^{\varepsilon /(\varepsilon-1)}
$$

where $\theta, \varepsilon, \omega_{i}>0$ and $\Sigma \omega_{i}=1$. Of course, if $\theta=1, v()=.\ln \phi($.$) and$ if $\varepsilon=1, \ln \phi()=.\sum_{i=1}^{m} \omega_{i} \ln c_{i}$. In the decentralized economy demand functions have constant price elasticity $-\varepsilon$ and unit income elasticity. With this utility function, (8) yields:

$$
\frac{p_{i} c_{i}}{p_{m} c_{m}}=\left(\frac{\omega_{i}}{\omega_{m}}\right)^{\varepsilon}\left(\frac{A_{m}}{A_{i}}\right)^{1-\varepsilon} \equiv x_{i} \quad \forall i .
$$

The new variable $x_{i}$ is the ratio of consumption expenditure on good $i$ to consumption expenditure on the manufacturing good and will prove useful in the subsequent analysis. The intuition behind this formula is in terms

\footnotetext{
0.

${ }^{5}$ The corresponding transversality condition is $\lim _{t \longrightarrow \infty} k \exp \left(-\int_{0}^{t}\left(F_{k}^{m}-\delta-\nu\right) d \tau\right)=$
} 
of price elasticities, given that all goods have unit income elasticity. The ratio of consumption expenditures is a weighted average of the ratio of the weights of each good in the utility function and of their relative prices. A higher price ratio $p_{i} / p_{m}$ raises the ratio of expenditure on good $i$ to good $m$ by one minus their common price elasticity.

We also define aggregate consumption expenditure and output per capita in terms of manufacturing:

$$
c \equiv \sum_{i=1}^{m} \frac{p_{i}}{p_{m}} c_{i} ; \quad y \equiv \sum_{i=1}^{m} \frac{p_{i}}{p_{m}} F^{i}
$$

Using static efficiency we derive:

$$
c=c_{m} X ; \quad y=A_{m} F(k, 1)
$$

where $X \equiv \sum_{i=1}^{m} x_{i}$.

\section{Structural change}

We define structural change as the state in which at least some of the labor shares are changing over time, i.e., $\dot{n}_{i} \neq 0$ for at least some $i$. We derive in the Appendix (Lemma A2) the employment shares:

$$
\begin{aligned}
n_{i}=\frac{x_{i}}{X}\left(\frac{c}{y}\right) \quad \forall i \neq m, \\
n_{m}=\frac{x_{m}}{X}\left(\frac{c}{y}\right)+\left(1-\frac{c}{y}\right) .
\end{aligned}
$$

The first term in the right side of (14) parallels the term in (13) and so represents the employment needed to satisfy the consumption demand for the manufacturing good. The second bracketed term is equal to the savings rate and represents the manufacturing employment needed to satisfy investment demand.

Conditions (13) and (14) drive our structural change results. To see the intuition behind them, note that by aggregation over all $i$, we obtain that in our economy the employment share used to produce consumption goods is equal to $c / y$, and the employment share used to produce capital goods is $1-c / y$. Conditions (13) and (14) state that the same holds for each sector $i$. From (10) and (12), the consumption expenditure share of each sector is $p_{i} c_{i} / p_{m} c=x_{i} / X$. So the employment share of consumption good $i$ is the consumption share of good $i$ multiplied by the employment share of total 
consumption. Equivalently, the employment share of consumption good $i$ is the average propensity to consume good $i: n_{i}=p_{i} c_{i} / p_{m} y$.

Condition (13) has the important implication that the growth rate of two sectors' relative employment depends only on the difference between the sectors' TFP growth rates and the elasticity of substitution between goods:

$$
\frac{\dot{n}_{i}}{n_{i}}-\frac{\dot{n}_{j}}{n_{j}}=(1-\varepsilon)\left(\gamma_{j}-\gamma_{i}\right) \quad \forall i, j \neq m
$$

But (8) implies that the growth rate of relative prices is:

$$
\frac{\dot{p}_{i}}{p_{i}}-\frac{\dot{p}_{j}}{p_{j}}=\gamma_{j}-\gamma_{i} \quad \forall i
$$

and so,

$$
\frac{\dot{n}_{i}}{n_{i}}-\frac{\dot{n}_{j}}{n_{j}}=(1-\varepsilon)\left(\frac{\dot{p}_{i}}{p_{i}}-\frac{\dot{p}_{j}}{p_{j}}\right) \quad \forall i, j \neq m
$$

Proposition 1 The rate of change of the relative price of good $i$ to good $j$ is equal to the difference between the TFP growth rates of sector $j$ and sector $i$. In sectors producing only consumption goods, relative employment shares grow in proportion to relative prices, with the factor of proportionality given by one minus the elasticity of substitution between goods. ${ }^{6}$

The dynamics of the individual employment shares satisfy:

$$
\begin{aligned}
\frac{\dot{n}_{i}}{n_{i}}= & \frac{\dot{c} / y}{c / y}+(1-\varepsilon)\left(\bar{\gamma}-\gamma_{i}\right) ; \quad \forall i \neq m \\
\frac{\dot{n}_{m}}{n_{m}}= & {\left[\frac{c / y}{c / y}+(1-\varepsilon)\left(\bar{\gamma}-\gamma_{m}\right)\right] \frac{(c / y)\left(x_{m} / X\right)}{n_{m}} } \\
& +\left(\frac{-c / y}{1-c / y}\right)\left(\frac{1-c / y}{n_{m}}\right)
\end{aligned}
$$

where $\bar{\gamma} \equiv \sum_{i=1}^{m}\left(x_{i} / X\right) \gamma_{i}$ is a weighted average of TFP growth rates, with the weight given by each good's consumption share.

Equation (18) gives the growth rate in the employment share of each consumption good as a linear function of its own TFP growth rate. The intercept and slope of this function are common across sectors but although the slope is a constant, the intercept is in general a function of time because both $c / y$ and $\bar{\gamma}$ are in general functions of time. Manufacturing, however, does not conform to this rule, because its employment share is a weighted

${ }^{6}$ All derivations and proofs, unless trivial, are collected in the Appendix. 
average of two components, one for the production of the consumption good, which conforms to the rule, and one for the production of capital goods, which behaves differently.

The properties of structural change follow immediately from (18) and (19). Consider first the case of equality in sectoral TFP growth rates, i.e., let $\gamma_{i}=\gamma_{m} \forall i$. Our economy in this case is one of balanced TFP growth, with relative prices remaining constant but with many differentiated goods. Because of the constancy of relative prices all consumption goods can be aggregated into one, so we effectively have a two-sector economy, one sector producing consumption goods and one producing capital goods. Structural change can still take place in this economy but only between the aggregate of the consumption sectors and the capital sector, and only if $c / y$ changes over time. If $c / y$ is increasing over time, the investment rate is falling and labor is moving out of the manufacturing sector and into the consumption sectors. Conversely, if $c / y$ is falling over time labor is moving out of the consumption sectors and into manufacturing. In both cases, however, the relative employment shares in consumption sectors are constant.

If $c / y$ is constant over time, structural change requires $\varepsilon \neq 1$ and different rates of sectoral TFP growth rates. It follows immediately from (16), (18) and (19) that if $\dot{c} / y=0, \varepsilon=1$ implies constant employment shares but changing prices. With constant employment shares faster-growing sectors produce relatively more output over time. Price changes in this case are such that consumption demands exactly match all the output changes due to the different TFP growth rates. But if $\varepsilon \neq 1$, prices still change as before but consumption demands are either too inelastic (in the case $\varepsilon<1)$ to match all the output change, or are too elastic $(\varepsilon>1)$ to be satisfied merely by the change in output due to TFP growth. So if $\varepsilon<1$ employment has to move into the slow-growing sectors and if $\varepsilon>1$ it has to move into the fast-growing sectors.

Proposition 2 If $\gamma_{i}=\gamma_{m} \forall i \neq m$, a necessary and sufficient condition for structural change is $\dot{c} / c \neq \dot{y} / y$. The structural change in this case is between the aggregate of consumption sectors and the manufacturing sector.

If $\dot{c} / c=\dot{y} / y$, necessary and sufficient conditions for structural change are $\varepsilon \neq 1$ and $\exists i \in\{1, . ., m-1\}$ s.t. $\gamma_{i} \neq \gamma_{m}$. The structural change in this case is between all sector pairs with different TFP growth rates. If $\varepsilon<1$ employment moves from the sector with the higher TFP growth rate to the sector with the lower TFP growth rate; conversely if $\varepsilon>1$. 
Proposition 2 for $\varepsilon<1$ confirms the structural change facts identified by Baumol et al. (1985). When demand is price inelastic, the sectors with the low productivity growth rate attract a bigger share of labor, despite the rise in their price. From the static efficiency results in (8) and (12) we find that the nominal output shares (defined as $p_{i} F^{i} / p_{m} y$ ) are equal to the employment shares in all sectors, and by (10) the nominal consumption shares are given by $x_{i} / X$, so the results obtained for employment shares also hold for nominal consumption and output shares. But real consumption growth satisfies

$$
\dot{c}_{i} / c_{i}-\dot{c}_{j} / c_{j}=\varepsilon\left(\gamma_{i}-\gamma_{j}\right) ; \quad \forall i, j,
$$

an expression also satisfied by real output shares $\forall i, j \neq m$.

A comparison of (15) with (20) reveals that a small $\varepsilon$ can reconcile the small changes in the relative real consumption shares with the large changes in relative nominal consumption shares found by Kravis et al. (1983). The authors concluded that their finding is evidence in favor of the technological explanation of structural change. More recently Daniel E. Sichel (1997) found the same pattern for relative output shares, and Falvey and Gemmell (1996) found that the real consumption share of services (a sector with low TFP growth rate) falls very gradually with income, both of which are consistent with our model when $\varepsilon<1$.

\section{Aggregate growth}

We now study the aggregate growth path of this economy, with the objective of finding a sufficient set of conditions that satisfy structural change as derived in the preceding section, and in addition satisfy Kaldor's stylized facts of aggregate growth. Recall that for the analysis of structural change we imposed a Hicks-neutral technology. It is well-known that with this type of technology, the economy can be on a steady state only if the production function is Cobb-Douglas. We therefore let $F\left(n_{i} k_{i}, n_{i}\right)=k_{i}^{\alpha} n_{i}, \alpha \in(0,1){ }^{7}$ With TFP in each sector growing at some rate $\gamma_{i}$, the aggregate economy will also grow at some rate related to the $\gamma_{i}$ s. The following Proposition derives the evolution of the aggregate economy:

\footnotetext{
${ }^{7}$ Daron Acemoglu and Veronica Guerrieri (2005) examined the implications of different capital intensities for economic growth and structural change. They show that capital deepening can cause both structural change and unbalanced growth. We examine in the Appendix the implications of different capital shares and a fixed factor for our model.
} 
Proposition 3 Given any initial $k(0)$, the equilibrium of the aggregate economy is a path for the pair $\{c, k\}$ that satisfies the following two differential equations:

$$
\begin{aligned}
\frac{\dot{k}}{k} & =A_{m} k^{\alpha-1}-\frac{c}{k}-(\delta+\nu), \\
\theta \frac{\dot{c}}{c} & =(\theta-1)\left(\gamma_{m}-\bar{\gamma}\right)+\alpha A_{m} k^{\alpha-1}-(\delta+\rho+\nu) .
\end{aligned}
$$

We define an aggregate balanced growth path such that aggregate output, consumption and capital grow at the same rate. It follows from Proposition 3 that a necessary condition for the existence of an aggregate balanced growth path is that the expression $(\theta-1)\left(\gamma_{m}-\bar{\gamma}\right)$ be a constant. To show this, let:

$$
(\theta-1)\left(\gamma_{m}-\bar{\gamma}\right) \equiv \psi \quad \text { constant }
$$

Define aggregate consumption and the capital-labor ratio in terms of efficiency units, $c_{e} \equiv c A_{m}^{-1 /(1-\alpha)}$ and $k_{e} \equiv k A_{m}^{-1 /(1-\alpha)}$ and let $g_{m} \equiv \gamma_{m} /(1-\alpha)$, the rate of labor-augmenting technological growth in the capital-producing sector.

The dynamic equations (21) and (22) become

$$
\begin{aligned}
\dot{c}_{e} / c_{e} & =\left[\alpha k_{e}^{\alpha-1}+\psi-(\delta+\nu+\rho)\right] / \theta-g_{m} \\
\dot{k}_{e} & =k_{e}^{\alpha}-c_{e}-\left(g_{m}+\delta+\nu\right) k_{e} .
\end{aligned}
$$

Equations (24) and (25) parallel the two differential equations in the control and state of the one-sector Ramsey economy, making the aggregate equilibrium of our many-sector economy identical to the equilibrium of the one-sector Ramsey economy when $\psi=0$, and trivially different from it otherwise. Both models have a saddlepath equilibrium and stationary solutions $\left(\hat{c}_{e}, \hat{k}_{e}\right)$ that imply balanced growth in the three aggregates. The capital-labor ratio is growing at the rate of growth of labor-augmenting technological progress in the sector that produces capital goods, $g_{m}$. Aggregate consumption and output deflated by the price of manufacturing goods are also growing at the same rate.

Proposition 2 and the requirement that $\psi$ be constant yield the important Proposition:

Proposition 4 Necessary and sufficient conditions for the existence of an aggregate balanced growth path with structural change are:

$$
\begin{aligned}
& \theta=1 \\
& \varepsilon \neq 1 ; \text { and } \exists i \in\{1, . ., m-1\} \text { s.t. } \gamma_{i} \neq \gamma_{m} .
\end{aligned}
$$


Recalling the definition of $\bar{\gamma}$ following equation (19), Proposition 3 implies that the contribution of each consumption sector $i$ to aggregate equilibrium is through its weight $x_{i}$ in $\bar{\gamma}$. Because each $x_{i}$ depends on the sector's relative TFP level, the weights here are functions of time. So $\bar{\gamma}$ cannot be constant during structural change and the only way that $\psi$ can be constant is through $\theta=1$, which yields $\psi=0$. In this case our aggregate economy in $c$ and $k$ becomes formally identical to the one-sector Ramsey economy with growth rate $\gamma_{m}$. There are two other conditions that give a constant $\psi$ and so yield balanced aggregate growth: $\gamma_{i}=\gamma_{m} \forall i$ or $\varepsilon=1$. But as Proposition 2 demonstrates neither condition permits structural change on the balanced growth path, where $c / y$ is constant.

Proposition 4 requires the utility function to be logarithmic in the consumption composite $\phi$, which implies an intertemporal elasticity of substitution equal to one, but be non-logarithmic across goods, which is needed to yield non-unit price elasticities. A noteworthy implication of Proposition 4 is that balanced aggregate growth does not require constant rates of growth of TFP in any sector other than manufacturing. Because both capital and labor are perfectly mobile across sectors, changes in the TFP growth rates of consumption-producing sectors are reflected in immediate price changes and reallocations of capital and labor across sectors, without effect on the aggregate growth path.

To give intuition for the logarithmic intertemporal utility function we recall that balanced aggregate growth requires that aggregate consumption be a constant fraction of aggregate wealth. With our homothetic utility function this can be satisfied either when the interest rate is constant or when consumption is independent of the interest rate. The relevant interest rate here is the rate of return to capital in consumption units, which is given by the net marginal product of capital, $\alpha y / k-\delta$, minus the change in the relative price of the consumption composite, $\gamma_{m}-\bar{\gamma}$. The latter is not constant during structural change. In the case $\varepsilon<1, \bar{\gamma}$ is falling over time (see Lemma A3 in the Appendix for proof), and so the real interest rate is also falling, and converging to $\alpha y / k-\delta$. With a non-constant interest rate the consumption-wealth ratio is constant only if consumption is independent of the interest rate, which requires a logarithmic utility function. ${ }^{8}$

\footnotetext{
${ }^{8}$ After re-examining the evidence, Robert Barro and Xavier Sala-i-Martin (2004, p.13) concluded, consistent with our model, "it seems likely that Kaldor's hypothesis of a roughly stable real rate of return should be replaced by a tendency for returns to fall
} 
Under the condition of Proposition 4 there is a steady-state characterized by aggregate balanced growth, in the sense that in this steady state the aggregate ratios are constant. In order to achieve this balance, the aggregates $c$ and $y$ are divided by manufacturing price, to conform to the aggregate $k$. If some other price index is used as deflator, the rate of growth of the aggregates is constant only if the rate of growth of the price index is constant, but of course the aggregate ratios are still constant. The published aggregate series studied by macroeconomists usually use an average price as deflator which does not have fixed weights. If the price index used to deflate national statistics is some $\tilde{p}$, the published real aggregate income is $y / \tilde{p}$. If the weights used to construct $\tilde{p}$ are the sector shares, $\tilde{p}$ changes during structural change. But because sector shares do not change rapidly over time, visually there is virtually nothing to distinguish the "stylized fact" of constant growth in reported per capita GDP with another "stylized fact" of constant growth in our per capita output measure. ${ }^{9}$

Next, we summarize the dynamics of employment shares along the aggregate balanced growth path.

Proposition 5 Let sector $l$ denote the sector with the smallest TFP growth rate when $\varepsilon<1$ or the sector with the biggest TFP growth rate when $\varepsilon>1$. On the aggregate balanced growth path, $n_{l}$ increases monotonically. Employment in the other sectors is either hump-shaped or declines monotonically. Asymptotically, the economy converges to an economy with

$$
n_{m}^{*}=\hat{\sigma}=\alpha\left(\frac{\delta+\nu+g_{m}}{\delta+\nu+\rho+g_{m}}\right) ; \quad n_{l}^{*}=1-\hat{\sigma}
$$

where $\hat{\sigma}$ is the savings rate along the aggregate balanced growth path.

Proposition 5 follows immediately from (18)-(19) and Lemma A3. Consider the case $\varepsilon<1$, the one for $\varepsilon>1$ following by a corresponding argument. For $\varepsilon<1$, sector $i$ expands if and only if its TFP growth rate is smaller than $\bar{\gamma}$, and contracts if and only if its growth rate exceeds it. But if $\varepsilon<1$, the weighted average $\bar{\gamma}$ is decreasing over time (see Lemma A3 in the Appendix). Therefore, the set of expanding sectors is shrinking

over some range as an economy develops." In our model it is converging from above to a positive value.

${ }^{9}$ Nicholas Kaldor (1961, p.178) spoke of a "steady trend rate" of growth in the "aggregate volume of production." In Ngai and Pissarides (2004, Fig.4) we plot our series of per capita real incomes and the published chain-weighted series for the United States since 1929, and show that they are virtually indistinguishable from each other. 
over time, as more sectors' TFP growth rates exceed $\bar{\gamma}$. This feature of the model implies that sectors with TFP growth rates below the initial $\bar{\gamma}$ exhibit a hump-shaped employment share, an implication that we believe is unique to our model. These employment shares first rise but once $\bar{\gamma}$ drops down to their own $\gamma_{i}$ they fall. ${ }^{10}$

In contrast to each sector's employment share, once the economy is on the aggregate balanced growth path output and consumption in each consumption sector grow according to

$$
\frac{\dot{F}^{i}}{F^{i}}=\frac{\dot{A}_{i}}{A_{i}}+\alpha \frac{\dot{k}_{i}}{k}+\frac{\dot{n}_{i}}{n_{i}}=\varepsilon \gamma_{i}+\alpha g_{m}+(1-\varepsilon) \bar{\gamma} .
$$

If $\varepsilon \leqslant 1$ the rate of growth of consumption and output in each sector is positive (provided $\gamma_{i} \geq 0$ ), and so sectors never vanish, even though their employment shares in the limit may vanish. If $\varepsilon>1$ the rate of growth of output may be negative in some low-growth sectors, and since by Lemma A $3 \bar{\gamma}$ is rising over time in this case, their rate of growth remains indefinitely negative until they vanish.

Finally, we examine briefly the implications of $\theta \neq 1$. When $\theta \neq 1$ balanced aggregate growth cannot coexist with structural change, because the term $\psi=(\theta-1)\left(\gamma_{m}-\bar{\gamma}\right)$ in the Euler condition (24) is a function of time. But as shown in the Appendix Lemma A3, $\bar{\gamma}$ is monotonic. As $t \rightarrow \infty, \psi$ converges to the constant $(\theta-1)\left(\gamma_{m}-\gamma_{l}\right)$, where $\gamma_{l}$ is the TFP growth rate in the limiting sector (the slowest or fastest growing consumption sector depending on whether $\varepsilon<$ or $>1$ ). Therefore, the economy with $\theta \neq 1$ converges to an asymptotic steady state with the same growth rate as the economy with $\theta=1$.

What characterizes the dynamic path of the aggregate economy when $\theta \neq 1$ ? By differentiation and using Lemma A3, we obtain

$$
\dot{\psi}=(\theta-1)(1-\varepsilon) \sum_{i=1}^{m}\left(x_{i} / X\right)\left(\gamma_{i}-\bar{\gamma}\right)^{2}
$$

which is of second-order compared with the growth in employment shares in (15), given that the $\gamma s$ are usually small numbers centered around 0.02 . Therefore, the rate of growth of the economy during the adjustment to the asymptotic steady state with $\theta \neq 1$ is very close to the constant growth

\footnotetext{
${ }^{10}$ Maddison (1980, p. 48) in his study of historical OECD data found a "shallow bell shape" for manufacturing employment for each of the 16 OECD countries, which can be reproduced by our model if the manufacturing TFP growth rate takes values between the TFP growth rates of agriculture and services.
} 
rate of the economy with $\theta=1$, despite ongoing structural change in both economies.

\section{Intermediate goods}

Our baseline model has no intermediate inputs and has only one sector producing capital goods. We now generalize it by introducing intermediate inputs and (in the next section) by allowing an arbitrary number of sectors to produce capital goods. The key difference between intermediate goods and capital goods is that capital goods are re-usable while intermediate goods depreciate fully after one usage. The motivation for the introduction of intermediate inputs is that many of the sectors that may be classified as consumption sectors produce in fact for businesses. Business services is one obvious example. Input-output tables show that a large fraction of output in virtually all sectors of the economy is sold to businesses. ${ }^{11}$

As in the baseline model, sectors are of two types. The first type produces perishable goods that are either consumed by households or used as intermediate inputs by firms. We continue referring to these sectors as consumption sectors. The second type of sector produces goods that can be used as capital. For generality's sake, we assume that the output of the capital-producing sector can also be processed into both consumption goods and intermediate inputs.

The output of consumption sector $i$ is now $c_{i}+h_{i}$, where $h_{i}$ is the output that is used as an intermediate good. Manufacturing output can be consumed, $c_{m}$, used as an intermediate input, $h_{m}$, or used as new capital, $\dot{k}$. We assume that all intermediate goods $h_{i}$ are used as an input into an aggregate CES production function $\Phi\left(h_{1}, \ldots, h_{m}\right)=\left[\sum_{i=1}^{m} \varphi_{i} h_{i}^{(\eta-1) / \eta}\right]^{\eta /(\eta-1)}$ that produces a single intermediate good $\Phi$, with $\eta>0, \varphi_{i} \geq 0$ and $\Sigma \varphi_{i}=1$. The production functions are modified to $F^{i}=A_{i} n_{i} k_{i}^{\alpha} q_{i}^{\beta}, \forall i$, where $q_{i}$ is the ratio of the intermediate good to employment in sector $i$ and $\beta$ is its input share, with $\alpha, \beta>0$ and $\alpha+\beta<1$. When $\beta=0$, we return to our baseline model. We show in the Appendix that a necessary and sufficient condition for an aggregate balanced growth path with structural change is $\eta=1$, i.e.

\footnotetext{
${ }^{11}$ According to input-output tables for the United States, in 1990 the percentage distribution of the output of two-digit sectors across three types of usage, final consumption demand, intermediate goods and capital goods was 43, 48 and 9 respectively. In virtually all sectors, however, a large fraction of the intermediate goods produced are consumed by the same sector.
} 
$\Phi($.$) should be Cobb-Douglas. { }^{12}$ When $\Phi($.$) is Cobb-Douglas, our central$ results from the baseline model carry through, with some modifications.

The aggregate equilibrium is similar to the one in the baseline model:

$$
\begin{aligned}
& \frac{\dot{c}}{c}=\alpha A k^{(\alpha+\beta-1) /(1-\beta)}-(\delta+\rho+\nu), \\
& \frac{\dot{k}}{\dot{k}}=(1-\beta) A k^{(\alpha+\beta-1) /(1-\beta)}-\frac{c}{k}-(\delta+\nu)
\end{aligned}
$$

where $A \equiv\left[A_{m}\left(\beta \Phi_{m}\right)^{\beta}\right]^{1 /(1-\beta)}$ and $\Phi_{m}$ is the marginal product of the manufacturing good in $\Phi$. The growth rate of $A$ is constant and equal to $\gamma=\gamma_{m}+\left(\beta \sum_{i=1}^{m} \varphi_{i} \gamma_{i}\right) /(1-\beta)$, where $\varphi_{i}$ is the input share of sector $i$ in $\Phi$. Therefore, we can define aggregate consumption and the aggregate capital-labor ratio in terms of efficiency units and obtain an aggregate balanced growth path with growth rate $\left(\gamma_{m}+\beta \sum_{i=1}^{m} \varphi_{i} \gamma_{i}\right) /(1-\alpha-\beta)$, which is the sum of labor-augmenting technological growth in the capitalproducing sector and a $\beta$ fraction of labor-augmenting technological growth in all sectors that produce intermediate goods. Recall the aggregate growth rate in the baseline model depended only on the TFP growth rate in manufacturing. In the extended model with intermediate goods, the TFP growth rates in all sectors contribute to aggregate growth.

The employment shares (13) and (14) are now modified to:

$$
\begin{aligned}
n_{i} & =\frac{x_{i}}{X}\left(\frac{c}{y}\right)+\varphi_{i} \beta ; \quad \forall i \neq m \\
n_{m} & =\left[\frac{x_{m}}{X}\left(\frac{c}{y}\right)+\varphi_{m} \beta\right]+\left(1-\beta-\frac{c}{y}\right) .
\end{aligned}
$$

For the consumption sectors, the extra term in (30) captures the employment required for producing intermediate goods. $\varphi_{i}$ is the share of sector $i$ 's output used for intermediate purposes and $\beta$ is the share of the aggregate intermediate input in aggregate output. For the manufacturing sector, the terms in the first bracket parallel those of the consumption sectors. The second term captures the employment share for investment purposes.

Our results on structural change now hold for the component of employment used to produce consumption goods, $\left(x_{i} / X\right)(c / y)$. The definition of $x_{i}$ and $X$ is the same as in the absence of intermediate goods. The contribution of intermediate goods to sectoral employment dynamics is the addition

\footnotetext{
${ }^{12}$ Nicholas Oulton (2001) claims that if there are intermediate goods, and if the elasticity of substitution between the intermediate goods and labor is bigger than 1, Baumol's "stagnationist" results could be overturned (in the absence of capital). No such possibility arises with Cobb-Douglas production functions.
} 
of the constant employment share $\varphi_{i} \beta$, with no impact on the other two components of employment. Following on from this, the asymptotic results in Proposition 5 are also modified. Asymptotically, the employment share used for the production of consumption goods still vanishes in all sectors except for the slowest growing one (when $\varepsilon<1$ ), but the employment share used to produce intermediate goods, $\varphi_{i} \beta$, survives in all sectors.

\section{Many capital goods}

In our second extension we allow an arbitrary number of sectors to produce capital goods. We study this extension with the baseline model without intermediate inputs.

We suppose that there are $\kappa$ different capital-producing sectors, each supplying the inputs into a production function $G$, which produces a capital aggregate that can be either consumed or used as an input in all production functions $F^{i}$. Thus, the model is the same as before, except that now the capital input $k_{i}$ is not the output of a single sector but of the production function $G$. The Appendix derives the equilibrium for the case of a CES function with elasticity $\mu$, i.e., when $G=\left[\sum_{j=1}^{\kappa} \xi_{m_{j}}\left(F^{m_{j}}\right)^{(\mu-1) / \mu}\right]^{\mu /(\mu-1)}$, where $\mu>0, \xi_{m_{j}} \geq 0$ and $F^{m_{j}}$ is the output of each capital goods sector $m_{j}$. $G$ now replaces the output of the "manufacturing" sector in our baseline model, $F^{m}$.

It follows immediately that the structural change results derived for the $m-1$ consumption sectors remain intact, as we have made no changes to that part of the model. But there are new results to derive concerning structural change within the capital-producing sectors. The relative employment shares across the capital-producing sectors satisfy:

$$
\begin{aligned}
n_{m_{j}} / n_{m_{i}} & =\left(\xi_{m_{j}} / \xi_{m_{i}}\right)^{\mu}\left(A_{m_{i}} / A_{m_{j}}\right)^{1-\mu} ; \quad \forall i, j=1, . ., \kappa \\
\frac{\dot{n}_{m_{j}}}{n_{m_{j}}}-\frac{\dot{n}_{m_{i}}}{n_{m_{i}}} & =(1-\mu)\left(\gamma_{m_{i}}-\gamma_{m_{j}}\right) ; \quad \forall i, j=1, . ., \kappa
\end{aligned}
$$

These equations parallel (13) and (15) of the baseline model and the intuition behind them is the same.

When there are many capital goods, the $A_{m}$ of the baseline model is replaced by $G_{m_{j}} A_{m_{j}}$ for each sector $m_{j}$, where $G_{m_{j}}$ denotes the sector's marginal product in the production of aggregate capital and $A_{m_{j}}$ is the sector's TFP level. This term measures the rate of return to capital in the $j$ th capital-producing sector, which is equal across all $\kappa$ sectors because 
of the free mobility of capital. In the Appendix we derive the aggregate growth rate:

$$
\gamma_{m}=\sum_{j=1}^{\kappa} \zeta_{j} \gamma_{m_{j}} ; \quad \zeta_{j} \equiv \xi_{m_{j}}^{\mu} A_{m_{j}}^{(\mu-1)} /\left(\sum_{i=1}^{\kappa} \xi_{m_{i}}^{\mu} A_{m_{i}}^{(\mu-1)}\right)
$$

which is a weighted average of TFP growth rates in all capital-producing sectors. The dynamic equations for $c$ and $k$ are the same as in the baseline model, given the new definition of $\gamma_{m}$.

If TFP growth rates are equal across all capital-producing sectors, $c$ and $k$ grow at a common rate in the steady state. But then all capital producing sectors can be aggregated into one, and the model reduces to one with a single capital-producing sector. If TFP growth rates are different across the capital-producing sectors and $\mu \neq 1$, there is structural change within the capital-producing sectors along the transition to the asymptotic state. Asymptotically, only one capital-producing sector remains. In the asymptotic state, $c$ and $k$ again grow at common rate, so there exists an asymptotic aggregate balanced growth path with only one capital-producing sector.

A necessary and sufficient condition for the coexistence of an aggregate balanced growth path and multiple capital-producing sectors with different TFP growth rates is $\mu=1$. The reason for this result is that a balanced aggregate path requires a constant $\gamma_{m}$, which is unattainable if the relative TFP levels in the capital-producing sectors are allowed to influence it. From (34), the influence of the productivity levels disappears only when $\mu=1$. The aggregate growth rate in this case is $\gamma_{m} /(1-\alpha)$ where $\gamma_{m}=$ $\sum_{j=1}^{\kappa} \xi_{m_{j}} \gamma_{m_{j}}$. Using (32), the relative employment shares across capitalproducing sectors are equal to their relative input shares in $G$. There is no structural change within the capital producing sectors, their relative employment shares remaining constant independently of their TFP growth rates.

The model with $\varepsilon<1$ and $\mu=1$ has clear contrasting predictions about the relation between the dynamics of sectoral employment shares and TFP growth (or relative prices). Sectors that produce primarily consumption goods should exhibit a well-defined linear relation between their employment share growth and their TFP growth rate; sectors that produce many intermediate goods should still have a positive linear relation, but less welldefined because of the constant term due to the production of intermediate goods. But sectors that produce primarily capital goods should exhibit no linear relation at all between their employment share growth and their 
relative TFP growth rate.

\section{Conclusion}

We have shown that different TFP growth rates across industrial sectors predict sectoral employment changes that are consistent with the facts if the substitutability between the final goods produced by each sector is low. Balanced aggregate growth requires in addition a logarithmic intertemporal utility function. Underlying the balanced aggregate growth there is a shift of employment away from sectors with high rate of technological progress towards sectors with low growth, and eventually, in the limit, all employment converges to only two sectors, the sector producing capital goods and the sector with the lowest rate of productivity growth. If the economy also produces intermediate goods the sectors that produce these goods also retain some employment in the limit, which is used to produce the intermediate goods.

Our results are consistent with the observation of simultaneous growth in the relative prices and employment shares of stagnant sectors such as community services, with the near-constancy of real consumption shares when compared with nominal shares. It is also consistent with the long-run evidence of Kuznets (1966) and Maddison (1980) concerning the decline of agriculture's employment share, the rise and then fall of the manufacturing share and the rise in the service share. The key requirement for these results is again a low substitutability between final goods. Of course, at a finer sector decomposition the elasticity of substitution between two goods may reasonably exceed unity; as for example between the output of the sector producing typewriters and the output of the sector producing electronic word processors. Our model in this case predicts that labor would move from the sector with low TFP growth to the one with the high TFP growth. The approach that we suggested for intermediate and many capital goods, namely the existence of subsectors that produce an aggregate that enters the utility or production function, is an obvious approach to the analysis of these cases. Within the subsectors there is structural change towards the high TFP goods but between the aggregates the flow is from high to low TFP sectors. 


\section{Appendix: Proofs}

Lemma A1 Equations (2), (5) and (7) imply equation (8).

Proof. Define $f(k) \equiv F(k, 1)$, omitting subscript $i$, (7) implies $F_{K}=$ $A f^{\prime}(k)$ and $F_{N}=A\left[f(k)-k f^{\prime}(k)\right]$. So $F_{N} / F_{K}=f(k) / f^{\prime}(k)-k$, which is strictly increasing in $k$. Hence, (5) implies $k_{i}=k_{m} \forall i \neq m$, and together with (2), results follow.

Lemma A2 $\forall i \neq m, n_{i}$ satisfy (13) and (18), and $n_{m}$ satisfy (14) and (19).

Proof. $n_{i}$ follows from substituting $F^{i}$ into (10), and $n_{m}$ is derived from (2). Given $\dot{x}_{i} / x_{i}=(1-\varepsilon)\left(\gamma_{m}-\gamma_{i}\right)$ and $\dot{X} / X=(1-\varepsilon)\left(\gamma_{m}-\bar{\gamma}\right)$, the result follows for $\dot{n}_{i}, i \neq m$. Using (2),

$$
\dot{n}_{m}=-\sum_{i \neq m} \dot{n}_{i}=-\frac{\dot{c} / y}{c / y}\left(1-n_{m}\right)-(1-\varepsilon)\left(\frac{c / y}{X}\right) \sum_{i \neq m} x_{i}\left(\bar{\gamma}-\gamma_{i}\right),
$$

so result follows for $\dot{n}_{m}$ by substituting $n_{m}$.

Proposition 3. Proof. Use (2) and (8) to rewrite (4) as

$$
\dot{k} / k=A_{m} k^{\alpha-1}\left(1-\sum_{i \neq m} n_{i}\right)-c_{m} / k-(\delta+\nu) .
$$

But $p_{i} / p_{m}=A_{m} / A_{i}$ and by the definition of $c$,

$$
\dot{k} / k=A_{m} k^{\alpha-1}-c / k-(\delta+\nu) .
$$

Next, $\phi$ is homogenous of degree one:

$$
\phi=\sum_{i=1}^{m} \phi_{i} c_{i}=\sum_{i=1}^{m} p_{i} c_{i} \phi_{m} / p_{m}=\phi_{m} c / p_{m}
$$

But $\phi_{m}=\omega_{m}\left(\phi / c_{m}\right)^{1 / \varepsilon}$ and $c=c_{m} X$, thus $\phi_{m}=\omega_{m}^{\varepsilon /(\varepsilon-1)} X^{1 /(\varepsilon-1)}$ and $v_{m}=$ $\phi^{-\theta} \phi_{m}=\left(\omega_{m}^{\varepsilon /(\varepsilon-1)} X^{1 /(\varepsilon-1)}\right)^{1-\theta} c^{-\theta}$, so (6) becomes (22).

Lemma A3 $d \bar{\gamma} / d t \lessgtr 0 \Leftrightarrow \varepsilon \lessgtr 1$.

Proof. Totally differentiating $\bar{\gamma}$ as defined in Proposition 3,

$$
\begin{aligned}
d \bar{\gamma} / d t & =\sum_{i=1}^{m}\left(x_{i} / X\right) \gamma_{i}\left(\dot{x}_{i} / x_{i}-\sum_{i=1}^{m} \dot{x}_{j} / X\right) \\
& =(1-\varepsilon) \sum_{i=1}^{m}\left(x_{i} / X\right) \gamma_{i}\left[\gamma_{m}-\gamma_{i}-\sum_{i=1}^{m}\left(x_{i} / X\right)\left(\gamma_{m}-\gamma_{j}\right)\right] \\
& =(1-\varepsilon)\left(\bar{\gamma}^{2}-\sum_{i=1}^{m}\left(x_{i} / X\right) \gamma_{i}^{2}\right)=-(1-\varepsilon) \sum_{i=1}^{m}\left(x_{i} / X\right)\left(\gamma_{i}-\bar{\gamma}\right)^{2}
\end{aligned}
$$

Since the summation term is always positive the result follows. 
Capital shares and fixed factors We now discuss the structural change results (equations (8) and (13)-(17)) when capital shares are different across sectors and there is a fixed factor of production in at least one sector. The production function is $F^{i}=A_{i} k_{i}^{\alpha_{i}} z_{i}^{1-\alpha_{i}-\beta_{i}} n_{i}$, where $\beta_{i}$ is labor share and $z_{i}=$ $Z_{i} / N_{i}$ is a fixed factor per unit of labor. Suppose $\alpha_{i}+\beta_{i}=1$ for $i \neq 1$, and $\alpha_{1}+\beta_{1}<1$, i.e. the fixed factor is used in sector 1 only. Static efficiency implies

$$
n_{i} / n_{j}=\left(\beta_{i} / \beta_{j}\right)\left(\omega_{i} / \omega_{m}\right)^{\varepsilon}\left(p_{i} / p_{j}\right)^{1-\varepsilon}, \forall i, j \neq m
$$

so the result that relative employment shares grow in proportion to relative prices, equation (17), is independent of different factor shares and the existence of a fixed factor . Our other results are modified as follows. The static efficiency condition (8) is replaced by

$$
\begin{aligned}
k_{i} & =\lambda_{i} k_{m}, \quad p_{i} / p_{m}=\left(A_{m} / A_{i}\right)\left(\varsigma_{m} / \varsigma_{i}\right) k_{m}^{\alpha_{m}-\alpha_{i}} z_{i}^{\alpha_{i}+\beta_{i}-1} ; \\
\lambda_{i} & \equiv \frac{\beta_{m} \alpha_{i}}{\beta_{i} \alpha_{m}}, \quad \varsigma_{i} \equiv \alpha_{i}^{\alpha_{i}} \beta_{j}^{1-\alpha_{i}} .
\end{aligned}
$$

Different capital shares add the term $\left(\alpha_{m}-\alpha_{i}\right) \dot{k}_{m} / k_{m}$ in (16). In a growth equilibrium with $k_{m}$ growing, lower $\alpha_{i}$ is another reason for higher relative price in sector $i$. Combining the relative price and relative employment equations, different capital shares add the term $(1-\varepsilon)\left(\alpha_{j}-\alpha_{i}\right) \dot{k}_{m} / k_{m}$ in (15). The existence of a fixed factor modifies (15) to $\left[1-\left(1-\alpha_{1}-\beta_{1}\right)(1-\varepsilon)\right] \frac{\dot{n}_{1}}{n_{1}}-\frac{\dot{n}_{j}}{n_{j}}=(1-\varepsilon)\left(\gamma_{j}-\gamma_{1}\right)+(1-\varepsilon)\left(\alpha_{j}-\alpha_{1}\right) \frac{\dot{k}_{m}}{k_{m}}$, $\forall j \neq m$. If $n_{1}$ is falling, then the presence of a fixed factor implies that $n_{1}$ falls at a faster rate. Finally, (13) and (14) are modified to

$$
n_{i}=\frac{x_{i}}{X} \frac{c}{y} \sum_{j} \frac{\beta_{i}}{\beta_{j}} n_{j}, \forall i \neq m ; \quad n_{m}=\frac{x_{m}}{X} \frac{c}{y} \sum_{j} \frac{\beta_{i}}{\beta_{j}} n_{j}+1-\frac{c}{y} \sum_{j} \frac{\beta_{i}}{\beta_{j}} n_{j},
$$

where $c=X c_{m}, y=A_{m} k_{m}^{\alpha_{m}} \sum_{i} \frac{\beta_{m} n_{i}}{\beta_{i}}$, and $x_{i}=\left(\frac{\omega_{i}}{\omega_{m}}\right)^{\varepsilon}\left(\varsigma_{i} k_{m}^{\alpha_{m}-\alpha_{i}} z_{i}^{\alpha_{i}+\beta_{i}-1} \frac{A_{m}}{A_{i}}\right)^{1-\varepsilon}$. The new system implies $n_{1}, \ldots, n_{m}$ can be solved simultaneously.

Intermediate goods $\forall i, F^{i} \equiv A_{i} n_{i} k_{i}{ }^{\alpha} q_{i}^{\beta}, \alpha, \beta \in(0,1), \alpha+\beta<1$. We have

$$
F^{m}=c_{m}+h_{m}+(\delta+\nu) k+\dot{k}, \quad F^{i}=c_{i}+h_{i}, \forall i \neq m .
$$

The planner's problem is similar to the baseline with (A1) replacing (3) and (4), $\left\{h_{i}, c_{i}, q_{i}\right\}_{i=1, . ., m}$ as additional controls and $\sum_{i=1}^{m} n_{i} q_{i}=\Phi\left(h_{1}, . ., h_{m}\right)$ as 
an additional constraint, where $\Phi$ is homogenous of degree one, $\Phi_{i}>0$ and $\Phi_{i i}<0$. The static efficiency conditions are:

$$
v_{i} / v_{m}=F_{K}^{m} / F_{N}^{i}=F_{N}^{m} / F_{N}^{i}=F_{Q}^{m} / F_{Q}^{i}=\Phi_{i} / \Phi_{m} ; \quad \forall i
$$

which implies $k_{i}=k, \quad q_{i}=\Phi, \quad$ and $p_{i}=A_{m} / A_{i} \quad \forall i$, so

$$
y=A_{m} k^{\alpha} \Phi^{\beta}, \quad \Phi=\sum_{i=1}^{m} \Phi_{i} h_{i}=\sum_{i=1}^{m} \Phi_{m} p_{i} h_{i}=\Phi_{m} h ; \quad h \equiv \sum_{i=1}^{m} p_{i} h_{i} .
$$

Optimal conditions for $h_{m}$ and $q_{m}$ imply $\beta \Phi_{m} A_{m} k^{\alpha} \Phi^{\beta-1}=1$, so $h=\beta y$ and (A1) is

$\dot{k}=A_{m} k^{\alpha} \Phi^{\beta}\left(1-\sum_{i \neq m} n_{i}\right)-h_{m}-c_{m}-(\delta+\nu) k=h(1-\beta) / \beta-c-(\delta+\nu) k$.

The dynamic efficiency condition is $-\dot{v}_{m} / v_{m}=\alpha A_{m} k^{\alpha-1} \Phi^{\beta}-(\delta+\rho+\nu)$, so

$$
\dot{c} / c=\alpha h /(\beta k)-(\delta+\rho+\nu), \quad \dot{k} / k=(1-\beta) h /(\beta k)-c / k-(\delta+\nu) .
$$

Constant $\dot{c} / c$ requires constant $h / k$ and constant $\dot{k} / k$ requires constant $c / k$. Thus, $\dot{h} / h$ must be constant. To derive constant $\dot{h} / h$, consider a CES $\Phi=$ $\left(\sum_{i=1}^{m} \varphi_{i} h_{i}^{(\eta-1) / \eta}\right)^{\eta /(\eta-1)}$, then (A2) implies $z_{i} \equiv p_{i} h_{i} / h_{m}=\left(\varphi_{i} / \varphi_{m}\right)^{\eta}\left(A_{m} / A_{i}\right)^{1-\eta}$, $\forall i$. So

$h=Z h_{m}, \quad \Phi_{m}=\varphi_{m}^{\eta /(\eta-1)} Z^{1 /(\eta-1)}, \quad \Phi=\left(\beta A_{m} k^{\alpha} \varphi_{m}^{\eta /(\eta-1)} Z^{1 /(\eta-1)}\right)^{1 /(1-\beta)}$,

where $Z \equiv \sum_{i=1}^{m} z_{i}$. Hence,

$$
h=\Phi / \Phi_{m}=\left(\beta A_{m} k^{\alpha}\right)^{1 /(1-\beta)}\left(\varphi_{m}^{\eta /(\eta-1)} Z^{1 /(\eta-1)}\right)^{\beta /(1-\beta)},
$$

and so

$$
(1-\beta) \dot{h} / h=\left(\gamma_{m}+\alpha \dot{k} / k\right)+\beta\left(\sum_{i=1}^{m}\left(z_{i} / Z\right) \gamma_{i}-\gamma_{m}\right),
$$

constant if $\sum_{i=1}^{m} z_{i} \gamma_{i}$ is constant. Given $\gamma_{i}$ differs across all $i$, constancy requires $\eta=1$, so

$$
\Phi=\prod_{i=1}^{m} h_{i}^{\varphi_{i}}, \quad Z=1 / \varphi_{m}, \quad z_{i}=\varphi_{i} / \varphi_{m} \quad \forall i .
$$

(A2) imply $\Phi=h_{m} \prod_{i=1}^{m}\left(z_{i} A_{i} / A_{m}\right)^{\varphi_{i}}$ and so $\Phi_{m}=\varphi_{m} \Phi / h_{m}=\prod_{i=1}^{m}\left(\varphi_{i} A_{i} / A_{m}\right)^{\varphi_{i}}$. But $\Phi=\left[\beta A_{m} k^{\alpha} \Phi_{m}\right]^{1 /(1-\beta)}$, so $h=\Phi / \Phi_{m}=\left(\beta A_{m} k^{\alpha}\right)^{1 /(1-\beta)} \Phi_{m}^{\beta /(1-\beta)}$. (A3) becomes

$$
\dot{c} / c+\delta+\rho+\nu=\alpha A k^{\alpha /(1-\beta)-1} ; \quad \dot{k}+c+(\delta+\nu) k=(1-\beta) A k^{\alpha /(1-\beta)},
$$


where $A \equiv\left[A_{m}\left(\beta \Phi_{m}\right)^{\beta}\right]^{1 /(1-\beta)}$. Define

$$
c_{e} \equiv c A^{-(1-\beta) /(1-\alpha-\beta)} ; \quad k_{e} \equiv k A^{-(1-\beta) /(1-\alpha-\beta)} ; \quad \gamma \equiv \dot{A} / A \text {. }
$$

We have $\gamma=\left[\gamma_{m}+\beta \sum_{i=1}^{m} \varphi_{i}\left(\gamma_{i}-\gamma_{m}\right)\right] /(1-\beta)=\gamma_{m}+\left(\beta \sum_{i=1}^{m} \varphi_{i} \gamma_{i}\right) /(1-\beta)$, and

$$
\begin{aligned}
\dot{c}_{e} / c_{e} & =\alpha k_{e}^{(\alpha+\beta-1) /(1-\beta)}-(\delta+\rho+\nu+g) ; \\
\dot{k}_{e} & =(1-\beta) k_{e}^{\alpha /(1-\beta)}-c_{e}-(\delta+\nu+g) k_{e}
\end{aligned}
$$

which imply the existence and uniqueness of an ABGP with growth rate,

$$
g \equiv(1-\beta) \gamma /(1-\alpha-\beta)=\left(\gamma_{m}+\beta \sum_{i=1}^{m} \varphi_{i} \gamma_{i}\right) /(1-\alpha-\beta)
$$

We obtain $n_{i}$ using $F^{i}=c_{i}+h_{i}, \forall i \neq m$, i.e.

$$
A_{i} n_{i} k^{\alpha} \Phi^{\beta} p_{i}=p_{i}\left(c_{i}+h_{i}\right)=x_{i} c_{m}+z_{i} h_{m}=c x_{i} / X+\varphi_{i} h .
$$

Substitute $p_{i}$ and $h$ to obtain $n_{i} y=c x_{i} / X+\varphi_{i} \beta y$, so (30) and (31) follow.

Many capital-producing sectors $\forall j, F^{m_{j}} \equiv A_{m_{j}} n_{m_{j}} k_{m_{j}}^{\alpha}$, which together produce good $m$ through

$$
G=\left[\sum_{j=1}^{\kappa} \xi_{m_{j}}\left(F^{m_{j}}\right)^{(\mu-1) / \mu}\right]^{\mu /(\mu-1)}, \quad \xi_{m_{j}} \geqslant 0, \mu>0, \sum_{j=1}^{\kappa} \xi_{m_{j}}=1 .
$$

The planner's problem is similar to the baseline model with (4) replaced by

$$
\dot{k}=G-c_{m}-(\delta+\nu) k
$$

and $\left(k_{m_{j}}, n_{m_{j}}\right)_{j=1, ., \kappa}$ as additional controls. The static efficiency conditions are

$$
F_{K}^{i} / F_{N}^{i}=F_{K}^{m_{j}} / F_{N}^{m_{j}}, \quad \forall i \neq m, \forall j
$$

so $k_{i}=k_{m_{j}}=k$. Also

$$
G_{m_{j}} / G_{m_{i}}=F_{K}^{m_{i}} / F_{K}^{m_{j}}=A_{m_{i}} / A_{m_{j}}, \forall i, j
$$

which implies $n_{m_{j}} / n_{m_{i}}=\left(\xi_{m_{j}} / \xi_{m_{i}}\right)^{\mu}\left(A_{m_{i}} / A_{m_{j}}\right)^{1-\mu}$ and grows at rate $(1-\mu)\left(\gamma_{m_{i}}-\gamma_{m j}\right)$. Let $n_{m} \equiv \sum_{j=1}^{\kappa} n_{m_{j}}$, we have $n_{m}=n_{m_{1}} \sum_{j=1}^{\kappa}\left(\xi_{m_{j}} / \xi_{m_{1}}\right)^{\mu}$ $\left(A_{m_{1}} / A_{m_{j}}\right)^{1-\mu}$. Next,

$$
p_{i}=v_{i} / v_{m}=A_{m} / A_{i}, \forall i \neq m,
$$


where $A_{m} \equiv G_{m_{1}} A_{m_{1}}$. Thus, $n_{i} / n_{j}$ and $p_{i} / p_{j}$ are the same as in the baseline.

To derive the aggregate equilibrium, note that $G=\sum_{j=1}^{\kappa} F^{m_{j}} G_{m_{j}}=$ $A_{m} k^{\alpha} n_{m}$, so $\dot{c} / c$ and $\dot{k} / k$ are the same as the baseline, so the equilibrium is the same as the baseline if $\gamma_{m} \equiv \dot{A}_{m} / A_{m}$ is constant, which we now derive. Given

$G_{m_{1}}=\xi_{m_{1}}\left(G / F^{m_{1}}\right)^{1 / \mu} ; \quad G / F^{m_{1}}=\left[\sum_{j=1}^{\kappa} \xi_{m_{j}}\left(A_{m_{j}} n_{m_{j}} /\left(A_{m_{1}} n_{m_{1}}\right)\right)^{(\mu-1) / \mu}\right]^{\mu /(\mu-1)}$, using the result on $n_{m_{j}} / n_{m_{1}}$ we have $G / F^{m_{1}}=\left[\sum_{j=1}^{\kappa} \xi_{m_{j}}^{\mu}\left(\xi_{m_{1}} A_{m_{1}}\right)^{1-\mu} A_{m_{j}}^{(\mu-1)}\right]^{\mu /(\mu-1)}$, thus $A_{m}=G_{m_{1}} A_{m_{1}}=\left[\sum_{j=1}^{\kappa} \xi_{m_{j}}^{\mu} A_{m_{j}}^{(\mu-1)}\right]^{1 /(\mu-1)}$, so

$$
\gamma_{m}=\sum_{j=1}^{\kappa} \zeta_{j} \gamma_{m_{j}}, \quad \zeta_{j} \equiv \xi_{m_{j}}^{\mu} A_{m_{j}}^{(\mu-1)} /\left(\sum_{j=1}^{\kappa} \xi_{m_{j}}^{\mu} A_{m_{j}}^{(\mu-1)}\right)
$$

constant if $(\mu-1) \sum_{j=1}^{\kappa} \zeta_{j}\left(\gamma_{m_{j}}-\gamma_{m}\right)^{2}=0$, i.e. if $(1) \gamma_{m_{i}}=\gamma_{m_{j}}, \forall i, j$, or $(2) \mu=1$. If (1) is true, the model reduces to only one capital-producing sector. Thus, coexistence of multiple capital-producing sectors and an ABGP requires (2), i.e., $G=\prod_{j=1}^{\kappa}\left(F^{m_{j}}\right)^{\xi_{j}}$ and $\gamma_{m}=\sum_{j=1}^{\kappa} \xi_{m_{j}} \gamma_{m_{j}}$. 


\section{References}

[1] Acemoglu, Daron and Guerrieri, Veronica, "Capital Deepening and Non-Balanced Economic Growth." Unpublished Paper, 2005.

[2] Barro, Robert J. and Sala-i-Martin, Xavier. Economic Growth. Second edition. Cambridge: The MIT Press, 2004.

[3] Baumol, William J. "Macroeconomics of Unbalanced Growth: The Anatomy of Urban Crisis." American Economic Review, 1967, 57(3): pp. 415-26.

[4] Baumol, William J.; Blackman, Sue Anne Batey and Wolff, Edward N. "Unbalanced Growth Revisited: Asymptotic Stagnancy and New Evidence." American Economic Review, 1985, 75(4), pp. 806-817.

[5] Caselli, Francesco and Coleman II, Wilbur John. "The U.S. Structural Transformation and Regional Convergence: A Reinterpretation." Journal of Political Economy, 2001, 109(3), pp. 584-616.

[6] Echevarria, Cristina. "Changes in Sectoral Composition Associated with Economic Growth." International Economic Review, 1997, 38(2), pp. 431-452.

[7] Falvey, Rodney E. and Gemmell, Norman. "Are Services Income Elastic? Some New Evidence." Review of Income and Wealth, 1996, 42(3), pp. 257-269.

[8] Foellmi, Reto and Zweimuller, Josef. "Structural Change, Engel's Consumption Cycles and Kaldor's Facts of Economic Growth." Unpublished Paper, 2005.

[9] Gollin, Douglas; Parente, Stephen and Rogerson, Richard. "The Role of Agriculture in Development." American Economic Review, 2002, 92(2), Papers and Proceedings, pp. 160-164.

[10] Kaldor, Nicholas. "Capital Accumulation and Economic Growth." in Friedrich A. Lutz and Douglas C. Hague, eds., The Theory of Capital. New York: St. Martin's Press, 1961, pp. 177-222.

[11] Kongsamut, Piyabha; Rebelo, Sergio and Xie, Danyang. "Beyond Balanced Growth." Review of Economic Studies, 2001, 68, pp. 869-882. 
[12] Kravis, Irving B.; Heston, Alan W. and Summers, Robert. "The Share of Services in Economic Growth," in F. Gerard Adams and Bert G. Hickman, eds., Global Econometrics: Essays in Honor of Lawrence R. Klein, Cambridge: MIT Press, 1983, pp.188-218.

[13] Kuznets, Simon. Modern Economic Growth: Rate, Structure, and Spread. New Haven: Yale University Press, 1966.

[14] Laitner, John. "Structural Change and Economic Growth". Review of Economic Studies, 2000, 67, pp. 545-561.

[15] Maddison, Angus, "Economic Growth and Structural Change in the Advanced Countries," in Irving Leveson and Jimmy W. Wheeler, eds., Western Economies in Transition: Structural Change and Adjustment Policies in Industrial Countries. London: Croom Helm, 1980, pp. 4165 .

[16] Ngai, L. Rachel and Pissarides, Christopher A. "Structural Change in a Multi-Sector Model of Growth." Centre for Economic Policy Research, CRPR Discussion Paper: No. 4763, 2004.

[17] Oulton, Nicholas (2001). "Must the Growth Rate Decline? Baumol's Unbalanced Growth Revisited." Oxford Economic Papers, 2001, 53, pp. 605-627.

[18] Sichel, Daniel E. "The Productivity Slowdown: Is a Growing Unmeasurable Sector the Culprit?" Review of Economics and Statistics, 1997, 79(3), pp. 367-370. 